PHYSICAL REVIEW FLUIDS 2, 029901(E) (2017)

\title{
Publisher's Note: Linear models for sound from supersonic reacting mixing layers [Phys. Rev. Fluids 1, 083801 (2016)]
}

P. Shivakanth Chary and Arnab Samanta

(Received 6 February 2017; published 10 February 2017)

DOI: 10.1103/PhysRevFluids.2.029901

This paper was published online on 21 December 2016 with an error in Fig. 9. Figure 9 has been replaced online as of 6 February 2017. 